\title{
CONTINUITY OF THE FENCHEL TRANSFORM OF CONVEX FUNCTIONS
}

\author{
KERRY BACK
}

\begin{abstract}
Given a separated dual system $\left(E, E^{\prime}\right)$, the Fenchel transform determines a pairing of the convex functions on $E$ with the convex functions on $E^{\prime}$. This operation is shown to have a continuity property. The result implies that the minimum set of a convex function varies in an upper-semicontinuous way with the function's conjugate. Several convergence concepts for convex functions are discussed. It is shown for each of the two most useful that the Fenchel transform is not a homeomorphism.
\end{abstract}

Let $\left(E, E^{\prime}\right)$ be a separated dual system. Denote by $\Gamma(E)$ the class of convex, $\sigma\left(E, E^{\prime}\right)$ lower-semicontinuous $\overline{\mathbf{R}}$-valued functions which do not take both the values $-\infty,+\infty$. These are the functions which are upper envelopes of collections of affine functions $x \rightarrow\left\langle x, x^{\prime}\right\rangle-a$, where $x^{\prime} \in E^{\prime}$ and $a \in \mathbf{R}$. The class $\Gamma\left(E^{\prime}\right)$ is defined symmetrically.

The Fenchel transform (conjugate) of $f \in \Gamma(E)$ is the function $f^{*} \in \Gamma\left(E^{\prime}\right)$ defined by $f^{*}\left(x^{\prime}\right)=\sup \left\{\left\langle x, x^{\prime}\right\rangle-f(x) \mid x \in E\right\}$. This determines a bijection of $\Gamma(E)$ onto $\Gamma\left(E^{\prime}\right)$, and, defining the conjugate of $f^{*} \in \Gamma\left(E^{\prime}\right)$ symmetrically, one has $f^{* *}=f$.

This paper studies the continuity of the Fenchel transform. The interest is in topologies on $\Gamma\left(E^{\prime}\right)$ for which the multifunction $f^{*} \rightarrow \operatorname{argmin} f^{*}$ has upper-semicontinuity properties, the ultimate objective being to provide conditions on sequences or nets of functions $f_{l} \in \Gamma(E)$ which, when satisfied, will ensure the convergence of the minimum sets of their conjugates.

The continuity of the Fenchel transform was first established by Wijsman [9] in finite-dimensional spaces. At the same time Walkup and Wets [7] showed that in reflexive Banach spaces the polarity operation for closed convex cones is an isometry, a fact which implies the continuity of the Fenchel transform in finitedimensional spaces (see Wets [8]). The continuity in reflexive Banach spaces was established by Mosco [3]. Joly [1] then obtained the same result and several generalizations by another argument.

Our point of departure is the work of Joly. In $\S 1$ we review his arguments, leading to a continuity theorem in Fréchet spaces.

In $\$ 2$ the concept of convergence in $\Gamma(E)$ will be strengthened and a continuity theorem will be proven without restrictions on the dual system $\left(E, E^{\prime}\right)$. The result

Received by the editors February 12, 1985.

1980 Mathematics Subject Classification (1985 Revision). Primary 46A55, 52A07. 
seems suitable for applications, but it is not symmetric. It will be demonstrated by examples that the convergence concept for $\Gamma(E)$ cannot be weakened nor that for $\Gamma\left(E^{\prime}\right)$ strengthened.

The results of convex analysis which will be used can be found in Rockafellar [6].

1. Topologies on $\Gamma(E)$ and $\Gamma\left(E^{\prime}\right)$. We briefly review the notation and results of Joly [1]. Let $\sigma$ be the weak topology $\sigma\left(E, E^{\prime}\right), \tau$ the Mackey topology $\tau\left(E, E^{\prime}\right)$, and $\sigma^{\prime}=\sigma\left(E^{\prime}, E\right), \tau^{\prime}=\tau\left(E^{\prime}, E\right)$. Joly defines a topology $\mathscr{T}_{\tau}$ on $\Gamma(E)$ in which a net $\left(f_{l}\right)$ converges to a point $f$ iff

$$
\inf _{x \in U} f(x) \geqslant \limsup \inf _{x \in U} f_{l}(x)
$$

for each $U \in \tau$. A topology $\mathscr{T}_{\tau^{\prime}}$ on $\Gamma\left(E^{\prime}\right)$ is defined symmetrically.

Denote by $\mathscr{T}_{\tau^{\prime}}^{*}$ the coarsest topology on $\Gamma(E)$ which renders continuous the Fenchel transform form $\Gamma(E)$ to $\left(\Gamma\left(E^{\prime}\right), \mathscr{T}_{\tau^{\prime}}\right)$. Convergence of a net $\left(f_{l}\right)$ in $\mathscr{T}_{\tau^{\prime}}^{*}$ is characterized by having

$$
\inf _{x^{\prime} \in U^{\prime}} f^{*}\left(x^{\prime}\right) \geqslant \limsup \inf _{x^{\prime} \in U^{\prime}} f_{l}^{*}\left(x^{\prime}\right)
$$

for each $U^{\prime} \in \tau^{\prime}$. Define $\mathscr{T}_{\tau}^{*}$ on $\Gamma\left(E^{\prime}\right)$ symmetrically, and denote by $\mathscr{T}_{\tau \tau^{\prime}}$ (resp. $\mathscr{T}_{\tau^{\prime} \tau}$ ) the least upper bound of the topologies $\mathscr{T}_{\tau}$ and $\mathscr{T}_{\tau^{\prime}}{ }^{*}$ (resp. $\mathscr{T}_{\tau^{\prime}}$ and $\mathscr{T}_{\tau}^{*}$ ).

Convergence of a net $\left(f_{l}\right)$ in $\mathscr{T}_{\tau \tau^{\prime}}$ and convergence of the conjugates $\left(f_{l}^{*}\right)$ in $\mathscr{T}_{\tau^{\prime} \tau}$ are both defined by having (1.1) and (1.2) hold simultaneously. Certainly the Fenchel transform is a homeomorphism of $\left(\Gamma(E), \mathscr{T}_{\tau \tau^{\prime}}\right)$ onto $\left(\Gamma\left(E^{\prime}\right), \mathscr{T}_{\tau^{\prime} \tau}\right)[\mathbf{1}$, Corollarie, p. 423].

This establishes the notation. The work to be done is to characterize the convergence of a net $\left(f_{l}\right)$ in $\mathscr{T}_{\tau \tau^{\prime}}$ in terms of directly verifiable conditions on the functions $f_{l}$ and to interpret the convergence of the functions $f_{l}^{*}$ in $\mathscr{T}_{\tau^{\prime} \tau}$.

Joly's insight is to introduce a topology $\mathscr{A}_{\tau^{\prime}}^{*}$ on $\Gamma(E)$ in which a net $\left(f_{l}\right)$ converges to a point $f$ iff

$$
\inf _{x^{\prime} \in U^{\prime}}\left(f+\psi_{K}\right)^{*}\left(x^{\prime}\right) \geqslant \limsup \inf _{x^{\prime} \in U^{\prime}}\left(f_{l}+\psi_{K}\right)^{*}\left(x^{\prime}\right)
$$

for each $U^{\prime} \in \tau^{\prime}$ and each weakly compact disk $K \subset E$ (the symbol $\psi_{K}$ denotes the function which takes the value 0 on $K$ and $+\infty$ elsewhere). A topology $\mathscr{A}_{\tau}^{*}$ on $\Gamma\left(E^{\prime}\right)$ is defined symmetrically.

Condition (1.3) is readily seen to be equivalent to having

$$
f(x) \leqslant \liminf _{i} f_{l_{i}}\left(x_{i}\right)
$$

for each $x \in E$, each subnet $\left(f_{l_{i}}\right)$ of the net $\left(f_{l}\right)$ and each net $\left(x_{i}\right)$ which is weakly convergent to $x$ and contained in a weakly compact disk [1, Remarque, p. 430].

Moreover, Joly observes that $\mathscr{A}_{\tau^{\prime}}^{*} \subset \mathscr{T}_{\tau^{\prime}}^{*}$ and $\mathscr{A}_{\tau}^{*} \subset \mathscr{T}_{\tau}^{*}$ [1, Proposition 7]. From the latter relation and the analogue of (1.4) we see that the set

$$
\left\{\left(f^{*}, x^{\prime}\right) \mid x^{\prime} \in \operatorname{argmin} f^{*}\right\} \cap\left[\Gamma\left(E^{\prime}\right) \times K\right]
$$

is $\left(\mathscr{T}_{\tau^{\prime} \tau} \times \sigma^{\prime}\right)$-closed for each weakly compact disk $K \subset E^{\prime}$. This upper-semicontinuity result seems suitable for applications since in practice one is likely to use the Banach-Alaoglu Theorem to verify the existence of a weak limit point of minimizing arguments for the functions $f_{l}^{*}$. Certainly it is satisfactory if $\left(E^{\prime}, \tau^{\prime}\right)$ is complete. 
It remains to characterize the convergence of the $f_{l}$ in $\mathscr{T}_{\tau \tau^{\prime}}$, specifically to state conditions on the $f_{l}$ which imply (1.2).

Denote by $\mathscr{A}_{\tau \tau^{\prime}}$ (resp. $\left.\mathscr{A}_{\tau^{\prime} \tau}\right)$ the least upper bound of the topologies $\mathscr{T}_{\tau}$ and $\mathscr{A}_{\tau^{\prime}}^{*}$ (resp. $\mathscr{T}_{\tau^{\prime}}$ and $\mathscr{A}_{\tau}^{*}$ ).

Joly observes that the relative $\mathscr{A}_{\tau^{\prime}}^{*}$ and $\mathscr{T}_{\tau^{\prime}}^{*}$ topologies coincide on subsets of $\Gamma(E)$ for which (the constant function) $+\infty$ is not an $\mathscr{A}_{\tau^{\prime}}^{*}$-adherent point [1, Proposition 8]. Thus a net converges in $\mathscr{T}_{\tau \tau^{\prime}}$ if it satisfies (1.1) and (1.4) and if its $\mathscr{A}_{\tau^{\prime}}^{*}$-adherence does not include $+\infty$. In certain cases one may deduce from convergence in $\mathscr{T}_{\tau}$, i.e. $(1.1)$, that the $\mathscr{A}_{\tau^{\prime}}^{*}$-adherence does not include $+\infty$ (the convergence in $\mathscr{A}_{\tau^{\prime}}^{*}$ has no implications in this regard since each $\mathscr{A}_{\tau^{\prime}}^{*}$ open set includes $+\infty$ ).

Proposition [1]. Suppose $E$ is a Fréchet space with dual $E^{\prime}$. Then a sequence $\left(f_{n}\right)$ from $\Gamma(E)$ converges to $f \not \equiv+\infty$ in $\mathscr{A}_{\tau \tau^{\prime}}$ if and only if the sequence $\left(f_{n}^{*}\right)$ converges to $f^{*}$ in $\mathscr{T}_{\tau^{\prime} \tau}$.

Proof. The claim is that convergence to $f \not \equiv+\infty$ in $\mathscr{A}_{\tau \tau^{\prime}}$ implies convergence in $\mathscr{T}_{\tau^{\prime}}{ }^{*}$. It suffices to show that convergence in $\mathscr{T}_{\tau}$ implies that the constant function $+\infty$ is not in the $\mathscr{A}_{\tau^{\prime}}^{*}$-adherence of the sequence $\left(f_{n}\right)$. Choosing $x$ such that $f(x)<\infty$, condition (1.1) implies the existence of a sequence $\left(x_{n}\right)$ which is $\tau$-convergent to $x$ and satisfies $\limsup _{n} f_{n}\left(x_{n}\right)<\infty$. Since $E$ is complete, the weakly closed disked hull of the sequence is weakly compact, and the result follows.

In other cases a different method will be necessary.

EXAmple 1 . Denote by $E$ the space of sequences $x=\left(\xi_{i}\right)$ with at most a finite number of nonzero terms, with the norm $\|x\|=\Sigma\left|\xi_{i}\right|$. Let $x_{n}$ be the sequence with all terms zero except the $n$ th, which is $1 / n$. Let $f_{n}=\psi_{\left\{x_{n}\right\}}$ and $f=\psi_{\{0\}}$. The sequence $\left(f_{n}\right)$ converges to $f$ in $\mathscr{T}_{\tau}$ and $\mathscr{A}_{\tau^{\prime}}^{*} ;$ in fact, (1.4) holds without the restriction that the net be contained in a weakly compact disk. Yet the constant function $+\infty$ is in the $\mathscr{A}_{\tau^{\prime}}^{*}$-adherence of the sequence $\left(f_{n}\right)$. This follows from the fact that each weakly compact disk in $E$ is finite dimensional (Kelley-Namioka [2, Problem 5.17.I]); so for such a disk $K$ we eventually have $f_{n}+\psi_{K} \equiv+\infty$.

2. A continuity Theorem and examples. We will say that a net $\left(f_{l}\right)$ is $M_{\sigma}$-convergent to $f$ in $\Gamma(E)$ if (1.4) holds for each $x \in E$, each subnet $\left(f_{l_{i}}\right)$ of the net $\left(f_{l}\right)$, and each net $\left(x_{i}\right)$ which is weakly convergent to $x$. This and the concept of $\mathscr{A}_{\tau^{*}}^{*}$-convergence as well are versions of a condition stated by Mosco [3] (for sequences and subsequences in a reflexive Banach space).

The net will be said to be $M_{\tau \sigma}$-convergent if it is $M_{\sigma}$-convergent and also convergent in the topology $\mathscr{T}_{\tau}$. It should be noted that this does not agree with the definition of Joly [1].

Example 1 shows that a sequence may be $M_{\tau \sigma}$-convergent but yet include $+\infty$ in its $\mathscr{A}_{\tau^{\prime}}^{*}$-adherence. We show by a direct argument that $M_{\tau \sigma}$-convergent nets are convergent in the topology $\mathscr{T}_{\tau \tau^{\prime}}$.

THEOREM. Suppose a net $\left(f_{l}, l \in L\right)$ is $M_{\tau o}$-convergent in $\Gamma(E)$ to $f \not \equiv+\infty$. Then the net $\left(f_{l}^{*}, l \in L\right)$ converges to $f^{*}$ in the topology $\mathscr{T}_{\tau^{\prime} \tau}$. 
Proof. We must show that (1.2) is satisfied. If $f \equiv-\infty$ then $f^{*} \equiv+\infty$ and (1.2) holds trivially. If $f \not \equiv-\infty$ then the $M_{\sigma}$-convergence implies that eventually the $f_{1}$ are different from the constant function $-\infty$. Furthermore, since $f \not \equiv+\infty$, the convergence in the topology $\mathscr{T}_{\tau}$ implies that eventually $f_{l} \not \equiv+\infty$. Hence we can and will assume that none of the $f_{l}$ nor $f$ is one of the constant functions $-\infty,+\infty$. This implies that the same is true of the $f_{l}^{*}$ and $f^{*}$, so we are dealing exclusively with what is termed in [6] "proper" convex functions.

Fix $U^{\prime} \in \tau^{\prime}$. There is no loss in assuming the existence of $x_{0}^{\prime}$ in $U^{\prime}$ satisfying $f^{*}\left(x_{0}^{\prime}\right)<\infty$. Let $K$ be a weakly compact disk in $E$ whose polar $K^{0} \equiv\left\{x^{\prime} \in\right.$ $\left.E^{\prime} \mid \psi_{K}^{*}\left(x^{\prime}\right) \leqslant 1\right\}$ satisfies $\left\{x_{0}^{\prime}\right\}-K^{0} \subset U^{\prime}$.

Step 1 . We will show that there is eventually $x_{l}^{\prime} \in E^{\prime}$ satisfying $\psi_{K}^{*}\left(x_{0}^{\prime}-x_{l}^{\prime}\right)<1$ and $f_{l}^{*}\left(x_{l}^{\prime}\right)<\infty$. Denote by $D$ (resp. $D_{l}$ ) the weak closure of the (convex) set $\left\{x^{\prime} \in E^{\prime} \mid f^{*}\left(x^{\prime}\right)<\infty\right\}$ (resp. $\left\{x^{\prime} \in E^{\prime} \mid f_{l}^{*}\left(x^{\prime}\right)<\infty\right\}$ ).

First we claim that

$$
\inf _{x \in E}\left[\psi_{D_{1}}^{*}(x)+\psi_{K}(x)-\left\langle x, x_{0}^{\prime}\right\rangle\right]=\sup _{x^{\prime} \in E^{\prime}}\left[-\psi_{D_{l}}\left(x^{\prime}\right)-\psi_{K}^{*}\left(x_{0}^{\prime}-x^{\prime}\right)\right] .
$$

To see this define $\phi: E \rightarrow \overline{\mathbf{R}}$ by

$$
\phi(u)=\inf _{x \in E}\left[\psi_{D_{1}}^{*}(x)+\psi_{K}(x-u)-\left\langle x-u, x_{0}^{\prime}\right\rangle\right] .
$$

The function $\phi$ is convex, lower-semicontinuous and never takes the value $-\infty$. Thus $\phi=\phi^{* *}$. The left-hand side of (2.1) is $\phi(0)$. Also for any $x^{\prime} \in E^{\prime}$ we have

$$
\begin{aligned}
\sup _{u \in E} & {\left[\left\langle u, x^{\prime}\right\rangle-\phi(u)\right]=\sup _{\substack{u \in E \\
x \in E}}\left[\left\langle u, x^{\prime}\right\rangle-\psi_{D_{l}}^{*}(x)-\psi_{K}(x-u)+\left\langle x-u, x_{0}^{\prime}\right\rangle\right] } \\
& =\sup _{x \in E}\left[\left\langle x, x^{\prime}\right\rangle-\psi_{D_{l}}^{*}(x)+\sup _{u \in E}\left\{\left\langle x-u, x_{0}^{\prime}-x^{\prime}\right\rangle-\psi_{K}(x-u)\right\}\right] \\
& \equiv \sup _{x \in E}\left[\left\langle x, x^{\prime}\right\rangle-\psi_{D_{l}}^{*}(x)+\psi_{K}^{*}\left(x_{0}^{\prime}-x^{\prime}\right)\right]=\psi_{D_{l}}(x)+\psi_{K}^{*}\left(x_{0}^{\prime}-x^{\prime}\right) .
\end{aligned}
$$

The right-hand side of $(2.1)$ is therefore $\phi^{* *}(0)$, so the equality holds.

Both sides of (2.1) are finite since the left-hand side is larger than $-\infty$ and the right-hand side is nonpositive. Hence it may be rewritten as

$$
\inf _{x \in K}\left[\psi_{D_{l}}^{*}(x)-\left\langle x, x_{0}^{\prime}\right\rangle\right]+\inf _{x^{\prime} \in D_{l}}\left[\psi_{K}^{*}\left(x_{0}^{\prime}-x^{\prime}\right)\right]=0 .
$$

Choose for each $l$ an $x_{l} \in K$ and an $x_{l}^{\prime} \in D_{l}$ satisfying

$$
\psi_{D_{l}}^{*}\left(x_{l}\right)-\left\langle x_{l}, x_{0}^{\prime}\right\rangle+\psi_{K}^{*}\left(x_{0}^{\prime}-x_{l}^{\prime}\right)<\frac{1}{2} \text {. }
$$

Denote by $M$ the set of $l \in L$ such that $\psi_{D_{l}}^{*}\left(x_{l}\right)-\left\langle x_{l}, x_{0}^{\prime}\right\rangle<-\frac{1}{2}$. It suffices to show that $M$ is not cofinal in $L$. Suppose for the sake of the argument that it is.

Since the net $\left(x_{l}, l \in M\right)$ is contained in $K$, there is a subnet $\left(x_{l_{i}}, i \in I\right)$ weakly convergent to an $x \in E$. We will show that $\left\langle x, x_{0}^{\prime}\right\rangle \leqslant \liminf _{i} \psi_{D_{l_{i}}}^{*}\left(x_{l_{i}}\right)$, contradicting the definition of $M$.

Notice first that $\left\langle x, x_{0}^{\prime}\right\rangle \leqslant \psi_{D}^{*}(x)$, since $x_{0}^{\prime} \in D$. Also, according to Rockafellar [5, Corollary 3c], $\psi_{D}^{*}(x)=\sup \{f(x+y)-f(y) \mid f(y)<\infty\}$. 
Choose an arbitrary $y \in E$ satisfying $f(y)<\infty$.

Denote by $\mathscr{U}$ the family of $\tau$-open sets containing $y$, and direct it by inclusion. Let $A$ denote the set of $(U, r, i) \in \mathscr{U} \times(0, \infty) \times I$ such that

$$
\inf _{z \in U} f_{l_{i}}(z) \leqslant f(y)+r^{-1} \text {. }
$$

By virtue of the convergence in $\mathscr{T}_{\tau}$ this inequality holds eventually in $i$ for each $(U, r)$. Thus $A$ is directed by the product order, and the projection of $A$ is cofinal in I.

For each $a=(U, r, i) \in A$ set $x_{a}=x_{l_{i}}, f_{a}=f_{l_{i}}, D_{a}=D_{l_{i}}$, and choose $y_{a} \in U$ in the following way: if $\inf \left\{f_{a}(z) \mid z \in U\right\}=-\infty$, let $f_{a}\left(y_{a}\right) \leqslant-r$; otherwise let $f_{a}\left(y_{a}\right) \leqslant \inf \left\{f_{a}(z) \mid z \in U\right\}+r^{-1}$. By this construction we have

$$
\limsup _{a} f_{a}\left(y_{a}\right) \leqslant \inf _{a} \max \left\{-r, f(y)+r^{-1}\right\} \leqslant f(y) .
$$

The net $\left(x_{a}, a \in A\right)$ is weakly convergent to $x$, and the net $\left(y_{a}, a \in A\right)$ is weakly convergent to $y$. We therefore obtain from the $M_{\sigma}$-convergence and (2.2) that

$$
f(x+y)-f(y) \leqslant \liminf _{a}\left[f_{a}\left(x_{a}+y_{a}\right)-f_{a}\left(y_{a}\right)\right] .
$$

By virtue of (2.2) we eventually have $f_{a}\left(y_{a}\right)<\infty$. Thus again applying [5, Corollary 3c] we obtain

$$
f(x+y)-f(y) \leqslant \liminf _{a} \psi_{D_{a}}^{*}\left(x_{a}\right) \equiv \liminf _{i} \psi_{D_{t_{i}}}^{*}\left(x_{l_{i}}\right),
$$

and since $y$ was chosen arbitrarily, this completes the argument.

Step 2. The function $x^{\prime} \rightarrow \psi_{K^{0}}\left(x_{0}^{\prime}-x^{\prime}\right)$ is $\tau^{\prime}$-continuous on $\left\{x^{\prime} \mid \psi_{K}^{*}\left(x_{0}^{\prime}-x^{\prime}\right)<\right.$ 1 ) so it follows from the preceding step and a version of Fenchel's Duality Theorem (see Rockafellar [4]) that eventually

$$
\inf _{x^{\prime} \in I^{\prime}}\left[f_{l}^{*}\left(x^{\prime}\right)+\psi_{K^{0}}\left(x_{0}^{\prime}-x^{\prime}\right)\right]=\max _{x \in E}\left[\left\langle x, x_{0}^{\prime}\right\rangle-\psi_{K^{0}}^{*}(x)-f_{l}(x)\right] .
$$

For each such $l$ choose $x_{l} \in E$ satisfying

$$
\inf _{x^{\prime} \in\left\{x_{0}^{\prime}\right\}-K^{0}} f_{l}^{*}\left(x^{\prime}\right)=\left\langle x_{l}, x_{0}^{\prime}\right\rangle-\psi_{K^{0}}^{*}\left(x_{l}\right)-f_{l}\left(x_{l}\right) .
$$

Given an arbitrary $\varepsilon>0$, denote by $M$ the set of such $l$ satisfying

$$
f^{*}\left(x_{0}^{\prime}\right)+\varepsilon \leqslant\left\langle x_{l}, x_{0}^{\prime}\right\rangle-\psi_{K^{0}}^{*}\left(x_{l}\right)-f_{l}\left(x_{l}\right) .
$$

Since the right-hand side of (2.3) majorizes $\inf \left\{f_{l}^{*}\left(x^{\prime}\right) \mid x^{\prime} \in U^{\prime}\right\}$, the proof will be complete if we show that $M$ is not cofinal in $L$. Suppose for the sake of the argument that it is.

We will deauce that the net $\left(x_{l}, l \in M\right)$ has a subnet $\left(x_{l_{i}}\right)$ which is weakly convergent to an $x \in E$. By virtue of the $M_{\sigma}$-convergence we must have

$$
\left\langle x, x_{0}^{\prime}\right\rangle-f(x) \geqslant \underset{i}{\limsup }\left[\left\langle x_{l_{i}}, x_{0}^{\prime}\right\rangle-f_{l_{i}}\left(x_{l_{i}}\right)\right] .
$$

But $f^{*}\left(x_{0}^{\prime}\right) \geqslant\left\langle x, x_{0}^{\prime}\right\rangle-f(x)$ by definition, and $\psi_{K^{0}}^{*} \geqslant 0$, so this will contradict the definition of $M$ and complete the proof. 
It is convenient to rewrite (2.3) as

$$
\psi_{K^{0}}^{*}\left(x_{l}\right) \leqslant\left\langle x_{l}, x_{0}^{\prime}\right\rangle-f_{l}\left(x_{l}\right)+s,
$$

where $s=-f^{*}\left(x_{0}^{\prime}\right)-\varepsilon \neq \pm \infty$ ( $s$ is less than $+\infty$ since $\left.f \not \equiv+\infty\right)$. Choose an $r>0$ and let $J_{r}=\left\{l \in M \mid\left\langle x_{l}, x_{0}^{\prime}\right\rangle-f_{l}\left(x_{l}\right)>r\right\}$. If $J_{r}$ is not cofinal in $M$ then, by virtue of (2.4), the net $\left(x_{l}, l \in M\right)$ is eventually within a weakly compact set, and we are done. Suppose conversely that $J_{r}$ is cofinal in $M$.

Fix $y \in E$ such that $f(y)<\infty$. As in Step 1 use the $\mathscr{T}_{\tau}$-convergence to construct a directed set $A$, an isotone map $v: A \rightarrow J_{r}$, and a net $\left(y_{a}, a \in A\right)$ which is $\tau$-convergent to $y$ and satisfies $\limsup _{a} f_{a}\left(y_{a}\right) \leqslant f(y)$, where we set $f_{a}=f_{v(a)}$.

Define $x_{a}=x_{v(a)}, \lambda_{a}=r \cdot\left[\left\langle x_{a}, x_{0}^{\prime}\right\rangle-f_{a}\left(x_{a}\right)\right]^{-1}$ and $z_{a}=\lambda_{a} x_{a}+\left(1-\lambda_{a}\right) y_{a}$. By the convexity of the $f_{a}$ we have

$$
\left\langle z_{a}, x_{0}^{\prime}\right\rangle-f_{a}\left(z_{a}\right) \geqslant r+\left(1-\lambda_{a}\right)\left[\left\langle y_{a}, x_{0}^{\prime}\right\rangle-f_{a}\left(y_{a}\right)\right] .
$$

Hence

$$
\liminf _{a}\left[\left\langle z_{a}, x_{0}^{\prime}\right\rangle-f_{a}\left(z_{a}\right)\right] \geqslant r+\min \left\{0,\left\langle y, x_{0}^{\prime}\right\rangle-f(y)\right\} .
$$

Observe now that $\psi_{K^{0}}^{*}\left(\lambda_{a} x_{a}\right)=\lambda_{a} \psi_{K^{0}}^{*}\left(x_{a}\right) \leqslant r+\lambda_{a} s$ by virtue of (2.4). Hence the net $\left(\lambda_{a} x_{a}, a \in A\right)$ is weakly precompact. There is therefore a subnet of the net $\left(z_{a}, a \in A\right.$ ) which is weakly convergent to some $z \in E$.

From the $M_{\sigma}$-convergence and (2.5) we obtain

$$
\left\langle z, x_{0}^{\prime}\right\rangle-f(z) \geqslant r+\min \left\{0,\left\langle y, x_{0}^{\prime}\right\rangle-f(y)\right\} .
$$

But $f^{*}\left(x_{0}^{\prime}\right) \equiv \sup \left\{\left\langle z, x_{0}^{\prime}\right\rangle-f(z) \mid z \in E\right\}<\infty$ by assumption and the choice of $y$ did not depend on $r$, so there must exist an $r$ such that $J_{r}$ is not cofinal in $M$.

It would be useful if $M_{\tau \sigma}$-convergence of functions in $\Gamma(E)$ implied $M_{\tau^{\prime} \sigma^{\prime}}$ convergence of the conjugates in $\Gamma\left(E^{\prime}\right)$, or if the Fenchel transform were a homeomorphism of $\left(\Gamma(E), \mathscr{A}_{\tau \tau^{\prime}}\right)$ onto $\left(\Gamma\left(E^{\prime}\right), \mathscr{A}_{\tau^{\prime} \tau}\right)$. Unfortunately, neither of these is true in general.

Example $1^{\prime}$. Define $E, f$ and the sequence $\left(f_{n}, n \in N\right)$ as in Example 1. We will show that the sequence $\left(f_{n}^{*}, n \in N\right)$ is not $M_{\sigma^{\prime}}$-convergent to $f^{*}$. Denote by $\mathscr{U}^{\prime}$ the family of disked $\sigma^{\prime}$ neighborhoods of the origin in $E^{\prime}$, and direct it by inclusion. Denote by $A$ the set of $\left(U^{\prime}, n\right) \in \mathscr{U}^{\prime} \times N$ for which there exists $x^{\prime} \in U^{\prime}$ satisfying $\left\langle x_{n}, x^{\prime}\right\rangle \leqslant-1$. For each $U^{\prime}$ we know that there is eventually such an $x^{\prime}$, since the polar of $U^{\prime}$ is finite dimensional. Hence $A$ is directed by the product order. Select for each $a=\left(U^{\prime}, n\right) \in A$ some $x_{a}^{\prime} \in U^{\prime}$ with the above property, and set $f_{a}^{*}=f_{n}^{*}$. The net $\left(f_{a}^{*}, a \in A\right)$ is a subnet of the sequence $\left(f_{n}^{*}, n \in N\right)$, the net $\left(x_{a}^{\prime}, a \in A\right)$ is weakly convergent to 0 , yet $\liminf _{a} f_{a}^{*}\left(x_{a}^{\prime}\right) \leqslant-1<0 \equiv f^{*}$.

ExAMPLE 2. Let $E$ be the dual of the space of sequences defined before, with a topology compatible with the pairing, so that $E^{\prime}$ is now the space of sequences. Define a sequence $\left(x_{n}\right)$ in $E$ by setting $\left\langle x_{n}, x^{\prime}\right\rangle=n \xi_{n}$ for each $x^{\prime}=\left(\xi_{1}, \xi_{2}, \ldots\right) \in$ $E^{\prime}$. Set $f=\psi_{\{0\}}$ and define $f_{n}\left(x_{n}\right)=-2 n, f_{n}(x)=+\infty$ for $x \neq x_{n}$. The Mackey and weak topologies on $E$ coincide since the weakly compact disks in $E^{\prime}$ are norm bounded finite-dimensional sets (see Kelley-Namioka [2, Problem 5.18.F]). Hence 
the sequence $\left(x_{n}\right)$ is $\tau$-convergent to 0 , and the sequence $\left(f_{n}\right)$ is therefore $\mathscr{T}_{\tau}$-convergent to $f$. It is also $\mathscr{A}_{\tau^{\prime}}^{*}$-convergent since the sequence $\left(x_{n}\right)$ eventually leaves the polar $K$ of any norm neighborhood of the origin in $E^{\prime}$, implying that $f_{n}+\psi_{K} \equiv+\infty$. However, the sequence $\left(f_{n}^{*}\right)$ does not converge to $f^{*}$ in the topology $\mathscr{T}_{\tau^{\prime}}:$ if $U^{\prime}$ is the unit ball in $E^{\prime}$ then

$$
\inf _{x^{\prime} \in U^{\prime}} f_{n}^{*}\left(x^{\prime}\right)=\inf _{x^{\prime} \in U^{\prime}}\left[\left\langle x_{n}, x^{\prime}\right\rangle+2 n\right]=n \nrightarrow 0 \equiv f^{*} .
$$

\section{REFERENCES}

1. J.-L. Joly, Une famille de topologies sur l'ensemble des fonctions convexes pour lesquelles la polarite est bicontinue. J. Math. Pures Appl. 52 (1973), 421-441.

2. J. L. Kelley and I. Namioka, Linear topological spaces, Springer-Verlag, New York, 1973.

3. U. Mosco, On the continuity of the Young-Fenchel transform, J. Math. Anal. Appl. 35 (1971), 518-535.

4. R. T. Rockafellar, Extension of Fenchel's duality theorem for convex functions, Duke Math. J. 33 (1966), 81-89.

5. Level sets and continuity of conjugate convex functions, Trans. Amer. Math. Soc. 123 (1966), $46-53$

6. Conjugate duality and optimization, CBMS-NSF Regional Conf. Ser. in Appl. Math., vol. 16, SIAM, Philadelphia, Pa., 1974.

7. D. Walkup and R. J.-B. Wets, Continuity of some convex-cone valued mappings, Proc. Amer. Math. Soc. 18 (1967), 229-235.

8. R. J.-B. Wets, Convergence of convex functions, variational inequalities and convex optimization problems, Variational Inequalities and Complementarity Problems (R. Cottle, F. Gianessi and J.-L. Lions, eds.), Wiley, London, 1980, pp. 375-403.

9. R. A. Wijsman, Convergence of sequences of convex sets, cones and functions. II, Trans. Amer. Math. Soc. 123 (1966), 32-45.

DePartment of Economics, NorthWESTERn University, EVANSTON, IllinOIS 60201 